\title{
Die Weiter- und Fortbildungswelt steht nicht still
}

Jedes Jahr liefert der Geschäftsbericht des Schweizerischen Instituts für ärztliche Weiter- und Fortbildung SIWF auf Zahlen und Fakten gestützt die Informationen, welche die geleistete Arbeit quantitativ und zusammenfassend beschreiben. Er bietet aber auch Gelegenheit, auf aktuelle Entwicklungen und Herausforderungen der ärztlichen Weiter- und Fortbildung hinzuweisen.

Die Zahlen dokumentieren, dass die Belastung und die Arbeit, die von den Mitarbeiterinnen und Mitarbeitern des Instituts geleistet werden, stetig zunehmen. Ihnen möchten wir zuallererst für ihr Engagement besonders herzlich danken. Sie setzen sich täglich dafür ein, die Gesuche um Titelerteilung, die Anträge auf Anerkennung als Weiterbildungsstätte, die Revisionen der Weiterbildungsprogramme und vieles mehr korrekt und speditiv zu bearbeiten. Das Gleiche gilt für die unzähligen Auskünfte auf Anfragen aus dem In- und Ausland und für die Organisation von mittlerweile um die
150 Visitationen, die ein zentrales Instrument der Qualitätssicherung darstellen.

Die Umsetzung der Weiter- und Fortbildungsordnung geht zwar mit vielen administrativen Aufgaben einher, doch der Kernauftrag des SIWF beschränkt sich keineswegs auf blosse Verwaltungsarbeiten. Die schnellen Veränderungen im Gesundheitswesen, der Strukturwandel an den Spitälern, die Knappheit zeitlicher und finanzieller Ressourcen und schnelle Entwicklungen in vielen Fachgebieten stellen Herausforderungen dar, die von Geschäftsleitung und Vorstand eine vorausschauende Aktualisierung des ärztlichen Bildungssystems verlangen. Dazu kommen Grossprojekte wie die Entwicklung eines zeitgerechten E-Logbuchs für alle Weiterzubildenden und der Ausbau der Fortbildungsplattform für alle aktiven Ärztinnen und Ärzte. Der vorliegende Bericht gibt einen Überblick über das gesamte Spektrum der Tätigkeiten des SIWF.

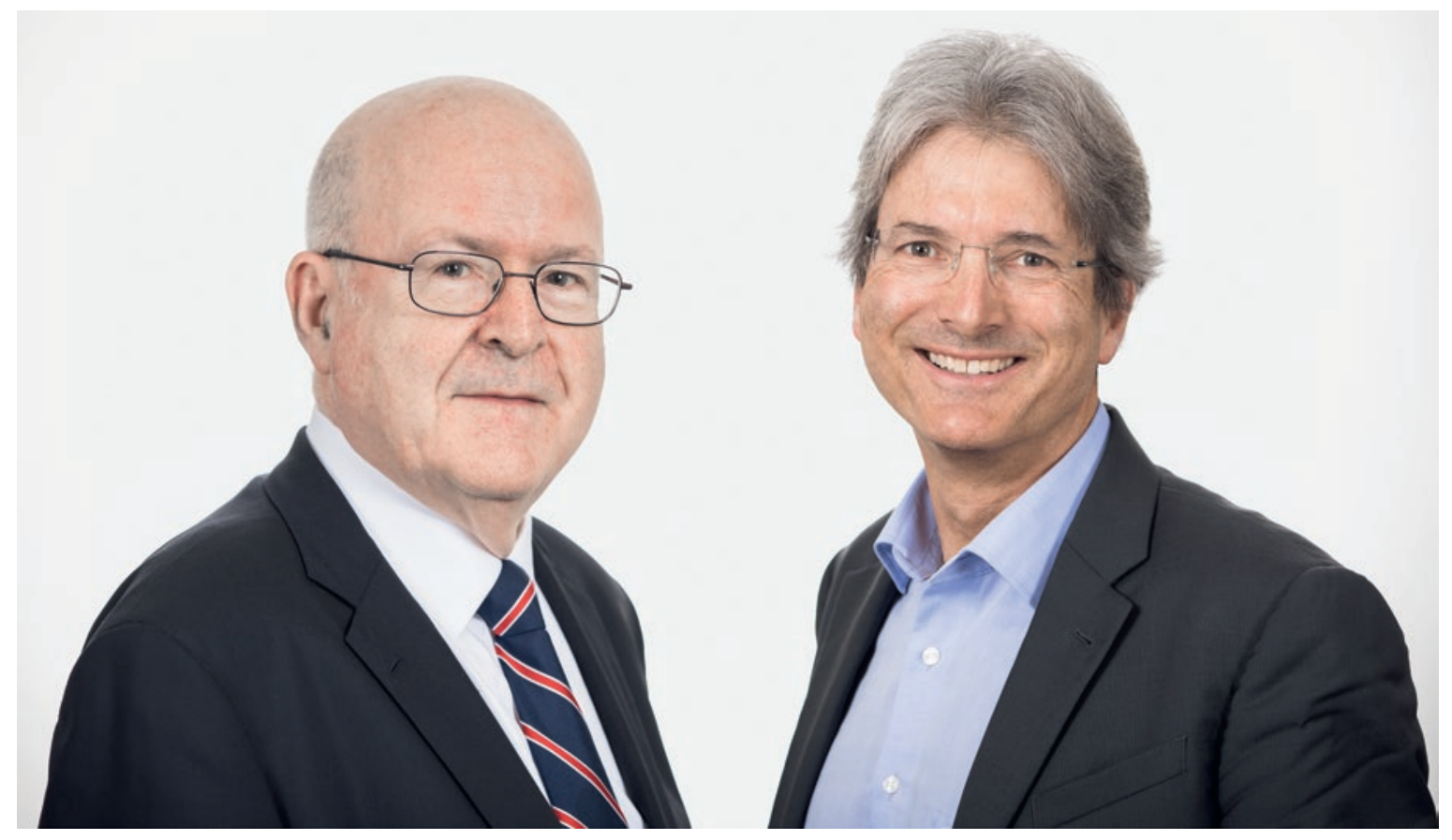

Dr. med. Werner Bauer, Präsident SIWF von 2010 bis Ende Januar 2021; Christoph Hänggeli, Rechtsanwalt, Geschäftsführer SIWF. 


\section{Ärztliche Bildung - Grundlage für die Qualität des Gesundheits- wesens von morgen}

\begin{abstract}
Die Ärztekammer hat als Ihre Nachfolgerin Frau PD Dr. med. et MME Monika Brodmann gewählt. Welche Gefühle weckt diese Stabübergabe in Ihnen?

Verschiedene Gefühle: Einmal, dass ich das schönste Amt ausüben durfte, das die schweizerische Ärzteschaft in meinen Augen zu vergeben hat. Dann, dass ich mich sehr freue, eine Nachfolgerin einführen zu dürfen, bei der ich das SIWF in besten Händen weiss. Und drittens, dass ich mich ohne besondere Vorbereitung, wie wir Ärzte sie auf die Pensionierung hin eigentlich empfehlen, nach dem «Volldampf im SIWF» neu orientieren muss.
\end{abstract}

\section{Warum das schönste Amt der Ärzteschaft?}

Weil es die ärztliche Bildung beinhaltet. Ich bin überzeugt, dass die gute Facharztweiterbildung von heute eine entscheidende Grundlage für die Qualität des Gesundheitswesens von morgen ist. Natürlich beruht diese nicht nur auf der Kompetenz der Ärzte. Viele andere Berufsgruppen müssen dazu beitragen, aber zu Prävention, Diagnostik, Therapie und Betreuung wer-

«Gute Facharztweiterbildung von heute ist eine entscheidende Grundlage für die Qualität des Gesundheitswesens von morgen.»

den die Ärztinnen und Ärzte stets einen unentbehrlichen Beitrag leisten. Dazu gehören Kenntnisse, Fähigkeiten und auch die persönliche Haltung - alles Bausteine der Weiterbildung, die deshalb anerkannt, mit genügenden Ressourcen gesichert und weiterentwickelt werden muss.

\section{Sie sind der zweite Präsident des Schweizerischen Instituts für ärztliche Weiter- und Fortbildung gewesen?}

Das trifft zu. Das SIWF wurde im Jahr 2009 gegründet. Der erste Präsident war Dr. med. Max Giger, der schon Präsident der Kommission für Weiter- und Fortbildung der FMH war, die durch das eigenständige SIWF abgelöst wurde. Das Präsidium habe ich im Juni 2010 übernommen, und meine Amtszeit hat sich um ein halbes Jahr verlängert, weil die Wahlärztekammer im Frühling ausfiel. In meine Amtszeit fielen zwei Akkreditierungen durch das Eidgenössische Departement des In- nern, das dem SIWF als verantwortlicher Organisation und den Fachgesellschaften das gute Wahrnehmen des staatlichen Auftrags der Facharztweiterbildung jeweils bestätigte.

\section{Wie waren Sie vorbereitet und was haben Sie angetroffen?}

Ich hatte bei meinem Amtsantritt schon ein «lampenfiebriges» Gefühl, auch wenn ich vorher in nationalen und europäischen Berufsorganisationen mit der ärztlichen Weiter- und Fortbildung intensiv konfrontiert war. Schnell habe ich realisiert: Unser Weiterbildungssystem mit über 100 Facharzttiteln, Schwerpunkten und Fähigkeitsausweisen leidet nicht unter einem Mangel an «Knacknussfragen», bei denen es oft um die Anerkennung von Titeln, von Weiterbildungsstätten, von ausländischer Weiterbildung oder um die Schaffung und um Revisionen von Weiterbildungsprogrammen geht. Sehr erleichtert wurde mir mein Start durch das engagierte und kompetente SIWF-Team, in erster Linie durch Christoph Hänggeli, den Geschäftsführer, und durch Barbara Linder, seine Stellvertreterin.

\section{Mit welchen Phasen der ärztlichen Bildung befasst sich das SIWF?}

Mit der Weiterbildung zur Fachärztin und zum Facharzt sowie mit der Fortbildung, die immer mehr auch zu einer beruflichen Weiterentwicklung wird. Im angelsächsischen Raum spricht man vom continuing professional development, weil die Ärzte in Zukunft vom Zeitpunkt des Facharztdiploms bis zu ihrer Pensionierung einen beträchtlichen Wandel der beruflichen Tätigkeit erleben werden und meistern müssen. Es geht bei der Fortbildung also um den Erhalt und auch den Neuerwerb von Kompetenzen. Dafür wird von uns Ärztinnen und Ärzten heute ein Nachweis erwartet, der mit dem SIWF-Fortbildungsdiplom online ohne grossen Aufwand erbracht werden kann.

Mit der Ausbildung hat das SIWF nur indirekt zu tun, indem die frisch diplomierten Ärztinnen und Ärzte sogleich zu unseren jüngsten Assistenzärztinnen und -ärzten werden und unsere Weiterbildungsstättenleiter davon ausgehen, ihnen mit gutem Gewissen erste Verantwortung übertragen zu können. Grundsätzlich müssen wir Aus-, Weiter- und Fortbildung als Kon- 


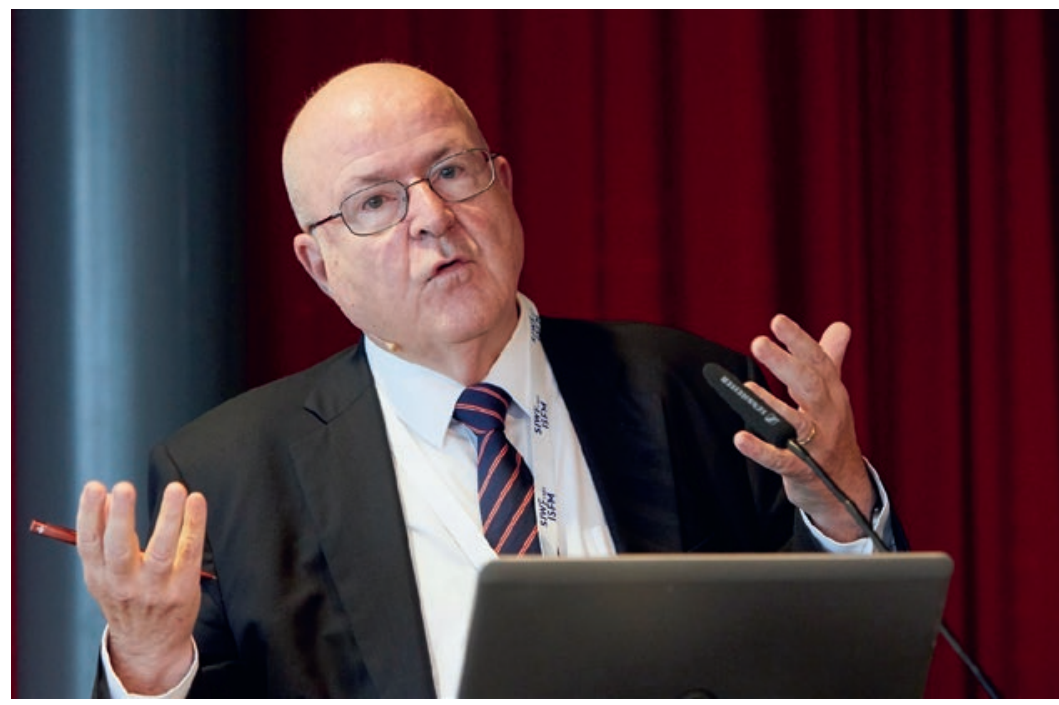

Werner Bauer war Präsident des SIWF von 2010 bis Ende Januar 2021.

tinuum sehen und gestalten. Wir führen deshalb alle Jahre eine Journée de réflexion durch, wo Vertreter der Fakultäten und des SIWF unter Einbezug von wichtigen Partnerinstitutionen (Bundesamt für Gesundheit, Gesundheitsdirektorenkonferenz, Verband Schweizerischer Assistenz- und Oberärztinnen und -ärzte, Schweizerische Akademie der Medizinischen Wissenschaften, H+ Die Spitäler der Schweiz) an der koordinierten Weiterentwicklung von Studium und Weiterbildung arbeiten.

\section{Haben Sie Neues einführen können?}

Neues einzuführen gehört zum Schönen eines jeden Amtes. Es ging mir dabei um Projekte mit dem Ziel, die Weiterbildung konkret zu unterstützen, und um das Positionieren des SIWF als schweizerisches Kompetenzzentrum für Weiter- und Fortbildung. Dafür schufen wir einen jährlichen Award für engagierte Weiterbildende und schrieben alle zwei Jahre eine «Projektförderung» aus. Für vier bis fünf von einer Jury ausgewählte Projekte, die direkt der Weiterbildung zugutekommen, stehen jeweils insgesamt rund 150000 Franken zur Verfügung. Die meisten eingereichten Gesuche waren von beachtenswerter Qualität, und die Auswahl fiel nicht leicht, wobei wir immer darauf achteten, dass ein Projekt nicht einen ausschliesslich lokalen Nutzen brachte.

FMH

Schweizerisches Institut

für ärztliche Weiter-

und Fortbildung SIWF

Nussbaumstrasse 29

Postfach

CH-3000 Bern 16

Tel. 0315030600

info[at]siwf.ch

\section{Sind nicht auch Veranstaltungen des SIWF dazugekommen?}

Ja, und zwar das MedEd-Symposium und die Faculty Development Workshops: Eine Institution wie das SIWF braucht in meinen Augen ein auch von aussen sichtbares Flaggschiff. Deshalb haben wir 2014 das erste MedEd-Symposium «Perspektiven der ärztlichen Bil- dung» durchgeführt, an dem seither jedes Jahr rund 200 Interessierte teilnehmen, die im Rahmen von Vorträgen, Seminaren, einem Podium oder einer Debatte und natürlich in den Pausen Informationen zu den verschiedensten Aspekten ärztlicher Bildung erhalten und diskutieren können. Die Evaluationen waren bis jetzt ausgesprochen positiv, was mich natürlich freut. Eine ganz besondere Erfolgsgeschichte stellen die Workshops dar, die wir mit Dozenten des Royal College of Physicians of London am Kantonsspital Luzern durchführen. Von meiner Tätigkeit in der European Federation of Internal Medicine her hatte ich Kontakte zum College, und weil teach the teachers eine wichtige Aufgabe unseres Instituts ist, habe ich den Versuch gewagt und die Londoner im Jahr 2012 erstmals eingeladen. Seither sind die Workshops schon zur Tradition geworden, und es finden jährlich zwei Serien statt. Themen sind zum Beispiel "Teaching in clinical settings", "How to deal with an underperforming trainee» oder "Leadership for doctors». Auch wenn die Kurse auf Englisch stattfinden, ist die Nachfrage ungebrochen und das Echo auch hinsichtlich der Anwendbarkeit des Gelernten hierzulande stets sehr gut. Wir haben nun begonnen, auch ein Team von Schweizer Instruktorinnen und Instruktoren aufzubauen, so dass in Ergänzung zu very British auch Workshops in den Landessprachen angeboten werden können.

\section{Was bleibt Ihrer Nachfolgerin denn noch zu tun?}

Vieles und Wichtiges. Das Gesundheitswesen, die ambulanten und stationären Institutionen und Strukturen sind im Umbruch, und davon bleibt die Weiterbildung nicht unberührt. Sie muss immer wieder ohne Abstrich an der Qualität den Rahmenbedingungen angepasst werden; sie muss im Hinblick auf Lernziele und Anerkennungskriterien die Entwicklungen in der Medizin abbilden; sie muss neue Methoden wie die Simulation und die Robotik integrieren, und sie muss auch didaktische Fortschritte wie das kompetenzbasierte Lernen mit den entrustable professional activities weiterentwickeln. Das sind grosse Aufgaben, auf die Monika Brodmann sich sicher freut. Ich wünsche ihr dafür von Herzen viel Erfolg und Befriedigung.

\section{Bildnachweis}

Tobias Schmid / SIWF (Bild Werner Bauer und Christoph Hänggeli) Heidi Fuchs / Medworld (Bild Werner Bauer)

\section{Vollständige Fassung SIWF-Geschäfts- bericht}

Die vollständige Fassung des SIWF-Geschäftsberichts inklusive des Berichts "SIWF Kompakt» und eines Rückblicks zur Geschäftsstelle des SIWF finden Sie auf der Website des SIWF unter: www.siwf.ch $\rightarrow$ Über das SIWF $\rightarrow$ Geschäftsberichte 


\section{$\underset{\text { SISFM }}{\text { SIWEMan }} \mid \mathbf{2 0 2 0}$}
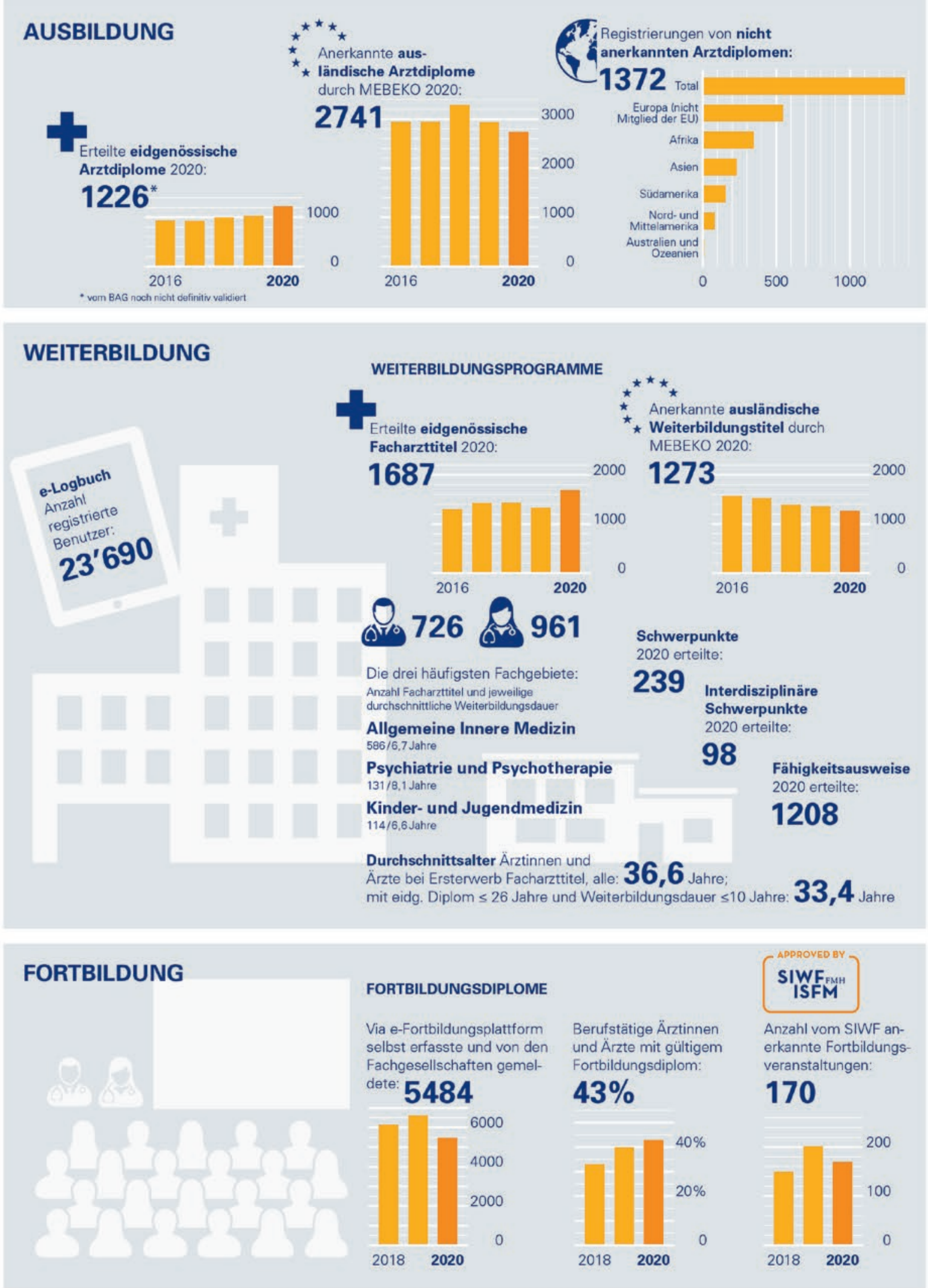


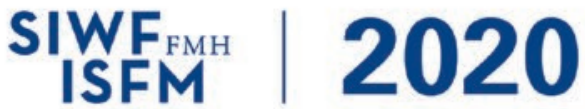

\section{FACHARZTTITEL}

Total erteilte Facharzttitel: 1687

davon an Ärzte mit CH-Diplom

(O) davon an Ärzte mit ausländischem Diplom

(die 10 häufigsten

Herkunftsländer)

\author{
1687
}

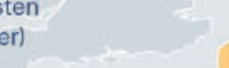

ASSISTENZ-

ÄRZTEUMFRAGE

An Umfrage teilgenommen:

\section{5}

Assistenzärztinnen und -ärzte (Rücklaufquote $70,10 \%$ )
Litauen

8

14

Rumänien

23

panien

12

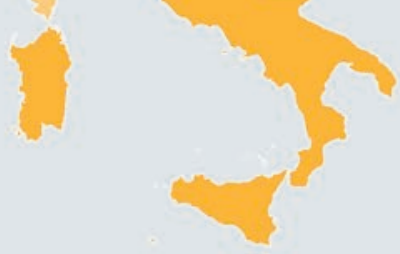

Griechenland

52

79 von 100 Arzten erachten den Beitrag der WBS zur Zusammenarbeit mit der Pflege als gross bis sehr gross.

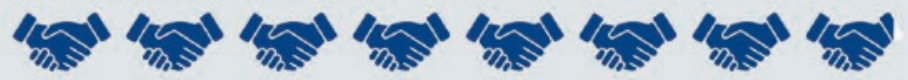

75 von 100 Ärztinnen und Ärrten würden die WBS voll und ganz, 19 von 100 Ärztinnen und Ärzte die WBS durchaus weiterempfehlen.
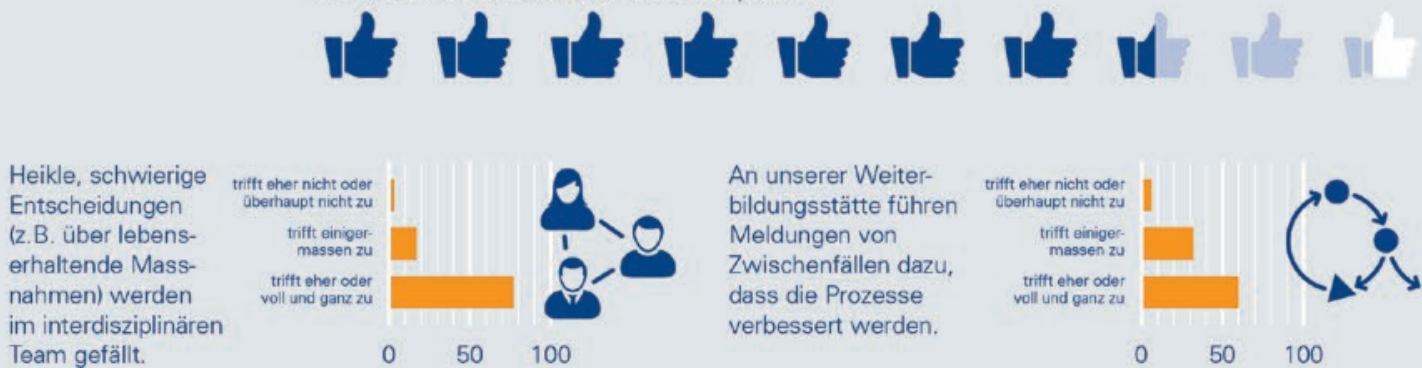